\title{
СИНТЕЗ И СТРОЕНИЕ СУЛЬФАТА МЕТИЛАММОНИЯ
}

\author{
(C) 2015 г. Р. Е. Хома*,**, В. О. Гельмбольдт***, В. Н. Баумер****, \\ А. Н. Пузан ${ }^{* * * * *}$, А. А. Эннан* \\ *Физико-химический институт защиты окружающей среды и человека \\ МОН Украины и НАН Украины, Одесса \\ **Одесский национальный университет имени И.И. Мечникова, Украина \\ ***Одесский национальный медицинский университет, Украина \\ ****НТК “Институт монокристаллов” НАН Украины, Харьков \\ *****Харьковский национальный университет им. В.Н. Каразина, Украина \\ E-mail: rek@onu.edu.ua \\ Поступила в редакцию 05.03.2015 г.
}

\begin{abstract}
Взаимодействие оксида серы(IV) с водным раствором метиламина приводит к получению "ониевого" сульфата состава $\left(\mathrm{CH}_{3} \mathrm{NH}_{3}\right)_{2} \mathrm{SO}_{4}$, охарактеризованного данными РСА, ИК-, масс-спектрометрии. Структура сульфата стабилизирована системой Н-связей типа $\mathrm{NH} \cdots \mathrm{O}$.
\end{abstract}

DOI: $10.7868 / \mathrm{S} 0044457 \mathrm{X} 15100104$

Метиламин (МА) является реагентом в синтезе лекарственных средств, инсектицидов, фунгицидов, красителей, ракетных топлив [1]. Метиламмонийный катион входит в состав солей с анионами различных неорганических кислот. Структурно охарактеризованы хлорид [2], дицитратоборат [3], бромид [4], гидрат тримолибдата [5], нитрат [6], перхлорат [7] и гексафторосиликат [8] метиламмония. Хотя сульфат метиламмония является коммерческим продуктом (CAS 33689-83-7), однако его кристаллическая структура ранее не была изучена. Авторы [9] привели лишь кристаллографические параметры указанного соединения, отнесенного к пр. гр. $P 2_{1} / c$.

В настоящей работе описаны результаты изучения строения и спектральных характеристик сульфата метиламмония $\left(\mathrm{CH}_{3} \mathrm{NH}_{3}\right)_{2} \mathrm{SO}_{4}$, выделенного в качестве продукта взаимодействия оксида серы(IV) с водным раствором МА в присутствии кислорода воздуха.

\section{ЭКСПЕРИМЕНТАЛЬНАЯ ЧАСТЬ}

Синтез сульфата метиламмония (I). В термостатируемую ячейку заливали 20 мл 40\%-ного водного раствора МА и пропускали через него газообразный $\mathrm{SO}_{2}$ при $0^{\circ} \mathrm{C}$ со скоростью 50 мл/мин до рН < $<1.0$. Полученный желтый маслообразный продукт выдерживали при $t<15^{\circ} \mathrm{C}$ на воздухе до испарения воды. Выделенный бесцветный кристаллический продукт I (15.43 г, выход 83.4\%) при температуре $>20^{\circ} \mathrm{C}$ превращается в ионную жидкость.
Содержание азота, углерода и водорода определяли с помощью элементного СHN-анализатора, серы - по Шенигеру [10]. ИК-спектры регистрировали на приборе Spectrum BX II FT-IR System (Perkin-Elmer) (диапазон 4000-650 см ${ }^{-1}$, образцы - в жидкостной кювете из $\left.\mathrm{BaF}_{2}\right)$; массспектры EI - на приборе MX-1321 (прямой ввод образца в источник, энергия ионизирующих электронов 70 эВ).

PCA выполнен на дифрактометре Xcalibur 3 (Oxford Diffraction Ltd), (МоK $K_{\alpha}$-излучение, графитовый монохроматор, CCD-детектор Sapphire-3). Pacшифровка и уточнение структуры проведены с использованием комплекса программ SHELX-97 [11]. Атомы водорода найдены из разностного синтеза Фурье и уточнены в изотропном приближении с общей тепловой поправкой.

Основные кристаллографические данные и результаты уточнения по структуре I: $\mathrm{C}_{2} \mathrm{H}_{12} \mathrm{~N}_{2} \mathrm{O}_{4} \mathrm{~S}$, моноклинная, $M_{r}=160.20$, пр. гр. $P 2_{1} / c, a=13.1442(7)$, $b=8.8370(5), c=13.5906(8) \AA, \beta=113.154(7)^{\circ}, V=$ $=1451.46(14) \AA^{3}$ при $T=293(2) \mathrm{K}, Z=8, \rho=$ $=1.466$ г $/ \mathrm{cm}^{3}, F_{000}=688$, кристалл $0.40 \times 0.30 \times 0.2 \mathrm{MM}$, $\mu=0.403 \mathrm{MM}^{-1}\left(\lambda\left(\mathrm{Mo}_{\alpha}\right)=0.71073 \AA\right)$, коэффициенты пропускания $T_{\min } / T_{\max }=0.2953 / 0.9237$; $-12 \leq h \leq 17,-11 \leq k \leq 11,-17 \leq l \leq 17$; $\omega$-сканирование при $2.94^{\circ} \leq \theta \leq 27.50^{\circ}, 6392$ измеренных отражений, из которых 3283 независимых $\left(R_{\mathrm{int}}=\right.$ $=0.0248)$ и 2380 наблюдаемых с $I_{h k l}>2 \sigma(I)$, полнота охвата $98.6 \%$; полноматричное уточнение 237 параметров по $F^{2}$ : окончательные показатели достоверности по наблюдаемым отражениям: 
Таблица 1. Координаты атомов $\left(\times 10^{4}\right)$ и эквивалентные тепловые поправки $\left(\AA^{2} \times 10^{3}\right)$ в структуре I

\begin{tabular}{|c|c|c|c|c|}
\hline Атом & $x / a$ & $y / b$ & $z / c$ & $U_{\text {ЭКв }}$ \\
\hline$S(1)$ & $10781(1)$ & $2616(1)$ & $4222(1)$ & $34(1)$ \\
\hline$S(2)$ & $5645(1)$ & $2658(1)$ & $4153(1)$ & $38(1)$ \\
\hline $\mathrm{O}(1)$ & $11060(1)$ & $1914(1)$ & $5282(1)$ & $47(1)$ \\
\hline $\mathrm{O}(2)$ & $11459(1)$ & $1930(2)$ & $3709(1)$ & $58(1)$ \\
\hline $\mathrm{O}(3)$ & $11025(1)$ & $4232(1)$ & $4362(1)$ & $60(1)$ \\
\hline $\mathrm{O}(4)$ & $9608(1)$ & $2405(2)$ & $3579(1)$ & $63(1)$ \\
\hline $\mathrm{O}(5)$ & 4589(1) & $1893(2)$ & $3897(1)$ & $63(1)$ \\
\hline $\mathrm{O}(6)$ & $6229(1)$ & $2024(2)$ & $3531(1)$ & $62(1)$ \\
\hline $\mathrm{O}(7)$ & $5436(1)$ & $4257(2)$ & $3890(1)$ & $79(1)$ \\
\hline $\mathrm{O}(8)$ & $6329(1)$ & $2494(2)$ & $5288(1)$ & $65(1)$ \\
\hline $\mathrm{N}(1)$ & $11199(1)$ & $4287(2)$ & $6754(1)$ & $43(1)$ \\
\hline $\mathrm{N}(2)$ & $8470(1)$ & $1211(2)$ & $4823(1)$ & $44(1)$ \\
\hline $\mathrm{N}(3)$ & 6929(1) & $-581(2)$ & 1141(1) & $45(1)$ \\
\hline $\mathrm{N}(4)$ & $5156(1)$ & 1291(2) & $6475(1)$ & $50(1)$ \\
\hline $\mathrm{C}(1)$ & $12297(2)$ & $4963(3)$ & $7028(2)$ & $61(1)$ \\
\hline $\mathrm{C}(2)$ & $8902(2)$ & 1791(2) & $5926(2)$ & $53(1)$ \\
\hline $\mathrm{C}(3)$ & $7482(2)$ & $29(3)$ & $2208(2)$ & $61(1)$ \\
\hline $\mathrm{C}(4)$ & $4018(2)$ & $1873(3)$ & $6083(2)$ & $61(1)$ \\
\hline $\mathrm{H}(1 \mathrm{~A})$ & $11123(16)$ & $3850(20)$ & $7316(17)$ & $72(1)$ \\
\hline $\mathrm{H}(1 \mathrm{~B})$ & $10685(16)$ & 4990(20) & $6499(16)$ & $72(1)$ \\
\hline $\mathrm{H}(1 \mathrm{C})$ & $11057(17)$ & $3560(20)$ & $6292(17)$ & $72(1)$ \\
\hline $\mathrm{H}(2 \mathrm{~A})$ & 8859(17) & $1650(20)$ & $4496(16)$ & $72(1)$ \\
\hline $\mathrm{H}(2 \mathrm{~B})$ & $7785(18)$ & $1440(20)$ & $4457(16)$ & $72(1)$ \\
\hline $\mathrm{H}(2 \mathrm{C})$ & $8556(17)$ & 180(30) & $4790(16)$ & $72(1)$ \\
\hline $\mathrm{H}(3 \mathrm{~A})$ & $7365(18)$ & $-1070(20)$ & 983(17) & $72(1)$ \\
\hline $\mathrm{H}(3 \mathrm{~B})$ & $6607(17)$ & 130(30) & 674(16) & $72(1)$ \\
\hline $\mathrm{H}(3 \mathrm{C})$ & $6433(17)$ & $-1300(20)$ & $1067(16)$ & $72(1)$ \\
\hline $\mathrm{H}(4 \mathrm{~A})$ & $5487(18)$ & $1680(20)$ & $6118(17)$ & $72(1)$ \\
\hline$H(4 B)$ & $5570(17)$ & $1700(20)$ & $7184(17)$ & $72(1)$ \\
\hline $\mathrm{H}(4 \mathrm{C})$ & $5193(16)$ & $260(20)$ & $6462(16)$ & $72(1)$ \\
\hline $\mathrm{H}(1 \mathrm{D})$ & $12362(17)$ & $5300(20)$ & $6398(17)$ & $72(1)$ \\
\hline $\mathrm{H}(1 \mathrm{E})$ & $12836(17)$ & $4280(20)$ & $7440(17)$ & $72(1)$ \\
\hline$H(1 F)$ & $12397(17)$ & $5700(30)$ & $7451(17)$ & $72(1)$ \\
\hline$H(2 D)$ & $8867(17)$ & $2890(30)$ & $5948(17)$ & $72(1)$ \\
\hline $\mathrm{H}(2 \mathrm{E})$ & $8445(18)$ & $1470(30)$ & $6198(17)$ & $72(1)$ \\
\hline $\mathrm{H}(2 \mathrm{~F})$ & $9630(18)$ & $1350(20)$ & $6240(17)$ & $72(1)$ \\
\hline $\mathrm{H}(3 \mathrm{D})$ & $7951(17)$ & $700(30)$ & $2248(17)$ & $72(1)$ \\
\hline $\mathrm{H}(3 \mathrm{E})$ & $7748(17)$ & $-720(30)$ & $2693(17)$ & $72(1)$ \\
\hline$H(3 F)$ & 6993(17) & $460(20)$ & $2422(16)$ & $72(1)$ \\
\hline $\mathrm{H}(4 \mathrm{D})$ & $4016(18)$ & $2980(30)$ & $6147(18)$ & $72(1)$ \\
\hline $\mathrm{H}(4 \mathrm{E})$ & $3689(18)$ & $1550(20)$ & $6499(17)$ & $72(1)$ \\
\hline $\mathrm{H}(4 \mathrm{~F})$ & $3564(17)$ & $1510(20)$ & $5434(17)$ & $72(1)$ \\
\hline
\end{tabular}

$R_{\mathrm{F}}=0.0394, w R^{2}=0.1035\left(R_{\mathrm{F}}=0.0582, w R^{2}=0.1165\right.$ по всем независимым отражениям), $S=0.941$, $\Delta \rho_{\min } / \Delta \rho_{\max }=-0.251 / 0.362$ э $/ \AA^{3}$.

\section{РЕЗУЛЬТАТЫ И ИХ ОБСУЖДЕНИЕ}

Состав соединения I установлен по данным элементного анализа.

\begin{tabular}{|c|c|c|c|c|}
\hline & $\mathrm{C}$ & $\mathrm{N}$ & $\mathrm{S}$ & $\mathrm{H}$ \\
\hline $\begin{array}{l}\text { Найдено, \%: } \\
\text { Для } \mathrm{C}_{2} \mathrm{H}_{12} \mathrm{~N}_{2} \mathrm{O}_{4} \mathrm{~S}\end{array}$ & 14.81 & 17.42 & 19.73; & 7.62 . \\
\hline числено, \%: & 15.00 & 17.49; & 20.03 & 7.55 . \\
\hline
\end{tabular}

Масс-спектр EI I: $[\mathrm{M}+\mathrm{H}]^{+}(m / z=32, I=15 \%)$; $[\mathrm{M}]^{+}(m / z=31, I=75 \%) ;\left[\mathrm{CH}_{2}=\mathrm{NH}_{2}\right]^{+}(m / z=30$, $I=100 \%)$. Характеристики продуктов фрагментации МА в масс-спектре I и табулированном массспектре МА [12] находятся в хорошем соответствии. В масс-спектре соединения I наблюдается характерная для 1-н-алкиламинов дефрагментация [13] с образованием иона $\left[\mathrm{CH}_{2}=\mathrm{NH}_{2}\right]^{+}$, пик которого имеет максимальную интенсивность.

Строение соединения I установлено методом РСА. Координаты атомов и эквивалентные тепловые поправки в структуре I даны в табл. 1. На рис. 1 показана схема нумерации атомов и эллипсоиды тепловых колебаний в независимой части ячейки. Длины связей и валентные углы, образуемые неводородными атомами в структуре, представлены в табл. 2. Как видно на рис. 1, в независимой части ячейки находятся четыре катиона $\mathrm{CH}_{3} \mathrm{NH}_{3}^{+}$и два иона $\mathrm{SO}_{4}^{2-}$. При упаковке этих базисных единиц в кристаллической структуре образуются водородные связи, характеристики которых даны в табл. 3. Все атомы водорода аммонийных групп участвуют в образовании водородных связей с атомами кислорода сульфат-ионов. Каждая из аммонийных групп атомов $\mathrm{N}_{1}, \mathrm{~N}_{2}, \mathrm{~N}_{3}, \mathrm{~N}_{4}$ образует водородные связи с тремя ближайшими сульфат-ионами, при этом одна из таких связей, образованных аммонийной группой атома $\mathrm{N}_{3}$, является “вилочной” (табл. 3). Система водородных связей, наблюдаемая в структуре I, является трехмерной, как видно из рис. 2.

Результаты анализа ИК-спектра I приведены в табл. 4. Отнесение колебаний метиламмония проведено с использованием данных [14-18]. Широкая полоса в ИК-спектре с четко выраженными тремя максимумами в области $3380-3000 \mathrm{~cm}^{-1}$ связана с колебаниями $v\left(\mathrm{NH}_{3}^{+}\right)$катиона метиламмония. Происхождение сложной полосы с максимумами при 2597, 2506, 2360 и $2342 \mathrm{~cm}^{-1}$ объясняется, повидимому, также наличием колебаний $v\left(\mathrm{NH}_{3}^{+}\right)$ (3000-2000 $\left.\mathrm{cm}^{-1}\right)$. В интервале частот $1600-1460 \mathrm{~cm}^{-1}$ 

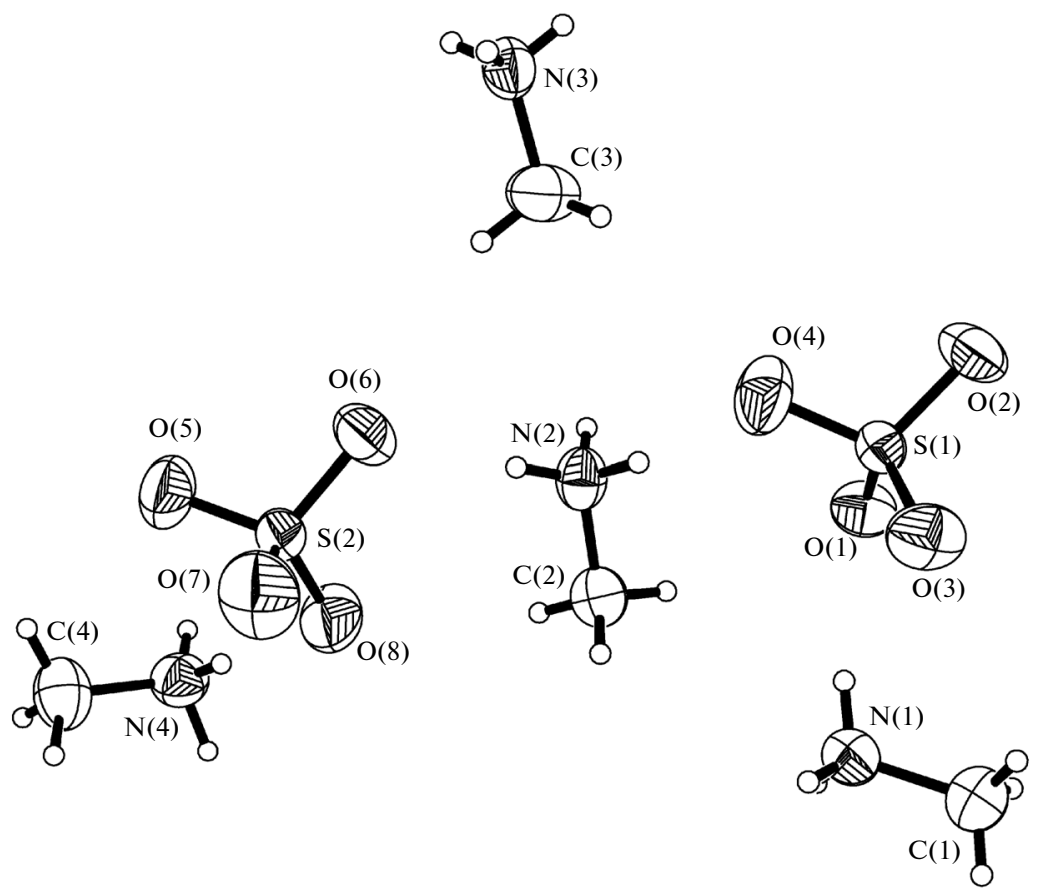

Рис. 1. Схема нумерации атомов и эллипсоиды тепловых колебаний в структуре I (уровень вероятности 50\%).

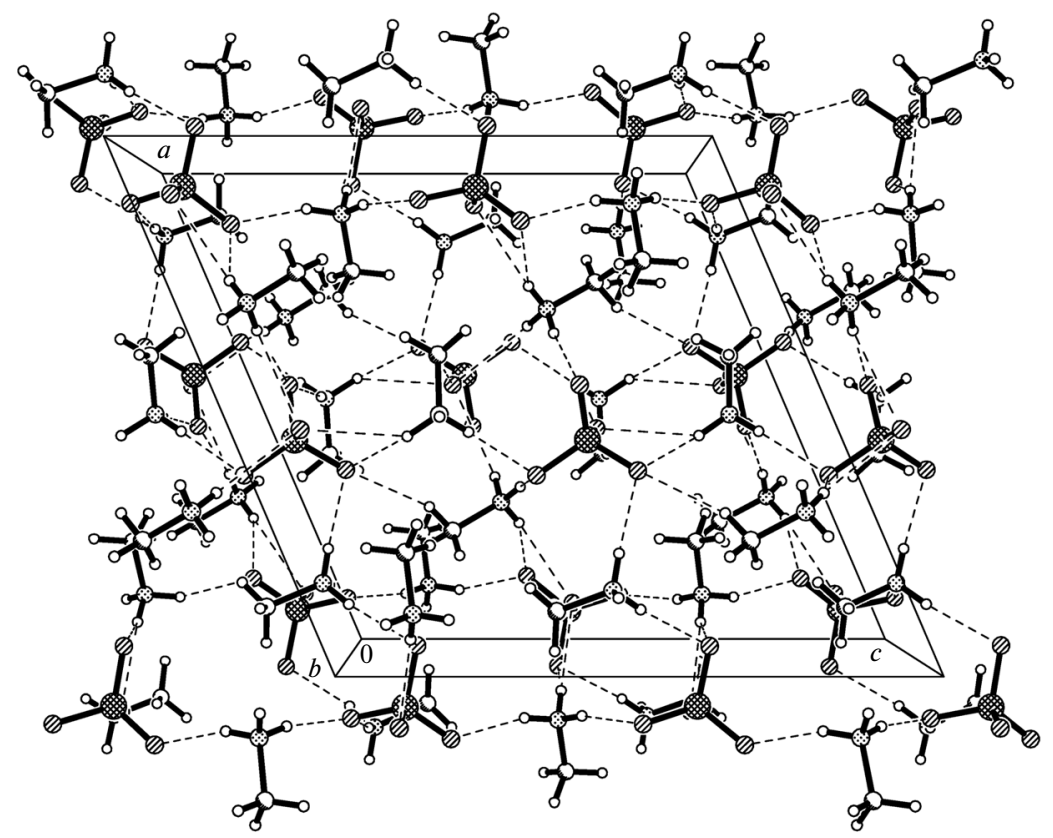

Рис. 2. Кристаллическая упаковка и система водородных связей в структуре І. Водородные связи изображены штриховыми линиями.

наблюдаются две интенсивные полосы при 1635 и $1521 \mathrm{~cm}^{-1}$, которые, очевидно, относятся к характерным для этой области ножничным деформационным колебаниям $\mathrm{NH}_{3}^{+}$-групп. Крутильные деформационные колебания аммонийных групп $\tau\left(\mathrm{NH}_{3}^{+}\right)$идентифицированы в виде малоинтенсивных полос при 1276 и $1239 \mathrm{~cm}^{-1}$.

Валентные колебания $v\left(\mathrm{~N}-\mathrm{CH}_{3}\right)$ проявляются при 2879 и $2783 \mathrm{~cm}^{-1}$, а ножничные деформационные $\delta\left(\mathrm{N}-\mathrm{CH}_{3}\right)-$ при 1466 и $1424 \mathrm{~cm}^{-1}$. 
Таблица 2. Длины связей и валентные углы в структуре I

\begin{tabular}{|c|c|c|c|c|c|}
\hline Связь & $d, \AA$ & Связь & $d, \AA$ & Связь & $d, \AA$ \\
\hline $\mathrm{S}(1)-\mathrm{O}(4)$ & $1.4540(13)$ & $\mathrm{S}(2)-\mathrm{O}(8)$ & $1.4556(13)$ & $\mathrm{N}(1)-\mathrm{C}(1)$ & $1.469(2)$ \\
\hline $\mathrm{S}(1)-\mathrm{O}(3)$ & $1.4601(13)$ & $\mathrm{S}(2)-\mathrm{O}(7)$ & $1.4569(15)$ & $N(2)-C(2)$ & $1.471(2)$ \\
\hline $\mathrm{S}(1)-\mathrm{O}(2)$ & $1.4621(13)$ & $\mathrm{S}(2)-\mathrm{O}(5)$ & $1.4578(13)$ & $\mathrm{N}(3)-\mathrm{C}(3)$ & $1.448(3)$ \\
\hline $\mathrm{S}(1)-\mathrm{O}(1)$ & $1.4755(11)$ & $\mathrm{S}(2)-\mathrm{O}(6)$ & $1.4585(13)$ & $N(4)-C(4)$ & $1.469(2)$ \\
\hline \multicolumn{2}{|c|}{ Угол } & $\omega$, град & \multicolumn{2}{|c|}{ Угол } & $\omega$, град \\
\hline \multicolumn{2}{|c|}{$\mathrm{O}(4) \mathrm{S}(1) \mathrm{O}(3)$} & $109.32(8)$ & & $109.55(9)$ \\
\hline \multicolumn{2}{|c|}{$\mathrm{O}(4) \mathrm{S}(1) \mathrm{O}(2)$} & $111.16(8)$ & \multicolumn{2}{|c|}{$\mathrm{O}(8) \mathrm{S}(2) \mathrm{O}(5)$} & $109.88(8)$ \\
\hline \multicolumn{2}{|c|}{$\mathrm{O}(3) \mathrm{S}(1) \mathrm{O}(2)$} & $108.86(8)$ & \multicolumn{2}{|c|}{$\mathrm{O}(7) \mathrm{S}(2) \mathrm{O}(5)$} & 108.73(9) \\
\hline \multicolumn{2}{|c|}{$\mathrm{O}(4) \mathrm{S}(1) \mathrm{O}(1)$} & $109.33(8)$ & \multicolumn{2}{|c|}{$\mathrm{O}(8) \mathrm{S}(2) \mathrm{O}(6)$} & $109.24(8)$ \\
\hline \multicolumn{2}{|c|}{$\mathrm{O}(3) \mathrm{S}(1) \mathrm{O}(1)$} & $109.05(7)$ & \multicolumn{2}{|c|}{$\mathrm{O}(7) \mathrm{S}(2) \mathrm{O}(6)$} & $108.81(10)$ \\
\hline \multicolumn{2}{|c|}{$\mathrm{O}(2) \mathrm{S}(1) \mathrm{O}(1)$} & $109.10(7)$ & \multicolumn{2}{|c|}{$\mathrm{O}(5) \mathrm{S}(2) \mathrm{O}(6)$} & $110.61(9)$ \\
\hline
\end{tabular}

Таблица 3. Характеристики водородных связей D-H...А в структуре I

\begin{tabular}{|c|c|c|c|c|c|}
\hline \multirow{2}{*}{ Контакт D-H...A } & \multicolumn{3}{|c|}{ Расстояние, $\AA$} & \multirow{2}{*}{ Угол DНА, град } & \multirow{2}{*}{ Координаты атома A } \\
\hline & $d(\mathrm{D}-\mathrm{H})$ & $d(\mathrm{H} \ldots \mathrm{A})$ & $d(\mathrm{D} \ldots \mathrm{A})$ & & \\
\hline $\mathrm{N}(1)-\mathrm{H}(1 \mathrm{~A}) \ldots \mathrm{O}(2)$ & $0.90(2)$ & $1.90(2)$ & $2.7607(19)$ & $161.4(18)$ & $x,-y+1 / 2, z+1 / 2$ \\
\hline $\mathrm{N}(4)-\mathrm{H}(4 \mathrm{~B}) \ldots \mathrm{O}(6)$ & $0.97(2)$ & $2.03(2)$ & $2.986(2)$ & $167.1(18)$ & $x,-y+1 / 2, z+1 / 2$ \\
\hline $\mathrm{N}(4)-\mathrm{H}(4 \mathrm{~B}) \ldots \mathrm{O}(7)$ & $0.97(2)$ & $2.54(2)$ & $3.193(2)$ & $124.7(16)$ & $x,-y+1 / 2, z+1 / 2$ \\
\hline $\mathrm{N}(1)-\mathrm{H}(1 \mathrm{~B}) \ldots \mathrm{O}(3)$ & $0.88(2)$ & $2.20(2)$ & $3.0112(19)$ & $153.6(19)$ & $-x+2,-y+1,-z+1$ \\
\hline $\mathrm{N}(1)-\mathrm{H}(1 \mathrm{~B}) \ldots \mathrm{O}(4)$ & $0.88(2)$ & $2.33(2)$ & $3.082(2)$ & $143.2(17)$ & $-x+2,-y+1,-z+1$ \\
\hline $\mathrm{N}(1)-\mathrm{H}(1 \mathrm{C}) \ldots \mathrm{O}(1)$ & $0.86(2)$ & $2.00(2)$ & $2.8538(19)$ & $168(2)$ & \\
\hline $\mathrm{N}(2)-\mathrm{H}(2 \mathrm{~A}) \ldots \mathrm{O}(4)$ & $0.89(2)$ & $1.98(2)$ & $2.864(2)$ & $170.8(19)$ & \\
\hline $\mathrm{N}(2)-\mathrm{H}(2 \mathrm{~B}) \ldots \mathrm{O}(6)$ & $0.86(2)$ & $2.00(2)$ & $2.8651(18)$ & $176(2)$ & \\
\hline $\mathrm{N}(2)-\mathrm{H}(2 \mathrm{C}) \ldots \mathrm{O}(1)$ & $0.92(2)$ & $1.93(2)$ & $2.8460(19)$ & $171.8(19)$ & $-x+2,-y,-z+1$ \\
\hline $\mathrm{N}(3)-\mathrm{H}(3 \mathrm{~A}) \ldots \mathrm{O}(2)$ & $0.81(2)$ & $2.28(2)$ & $3.005(2)$ & $150(2)$ & $-x+2, y-1 / 2,-z+1 / 2$ \\
\hline $\mathrm{N}(3)-\mathrm{H}(3 \mathrm{~A}) \ldots \mathrm{O}(3)$ & $0.81(2)$ & $2.36(2)$ & $3.029(2)$ & $141.1(19)$ & $-x+2, y-1 / 2,-z+1 / 2$ \\
\hline $\mathrm{N}(3)-\mathrm{H}(3 \mathrm{~B}) \ldots \mathrm{O}(8)$ & $0.87(2)$ & $2.16(2)$ & $2.948(2)$ & $149.3(19)$ & $x,-y+1 / 2, z-1 / 2$ \\
\hline $\mathrm{N}(3)-\mathrm{H}(3 \mathrm{~B}) \ldots \mathrm{O}(7)$ & $0.87(2)$ & $2.37(2)$ & $3.139(2)$ & $147.6(19)$ & $x,-y+1 / 2, z-1 / 2$ \\
\hline $\mathrm{N}(4)-\mathrm{H}(4 \mathrm{~A}) \ldots \mathrm{O}(8)$ & $0.84(2)$ & $2.00(2)$ & $2.840(2)$ & $177(2)$ & \\
\hline $\mathrm{N}(4)-\mathrm{H}(4 \mathrm{C}) \ldots \mathrm{O}(5)$ & $0.91(2)$ & $2.01(2)$ & $2.901(2)$ & $164.1(19)$ & $-x+1,-y,-z+1$ \\
\hline
\end{tabular}

Полоса при $969 \mathrm{~cm}^{-1}$ может быть отнесена к смешанным валентно-деформационным колебаниям $v_{\mathrm{s}}\left(\mathrm{SO}_{4}^{2-}\right), \tau\left(\mathrm{NH}_{3}^{+}\right)$и $\rho\left(\mathrm{CH}_{3}\right)$. Деформационные колебаний $\delta_{\text {as }}\left(\mathrm{SO}_{4}^{2-}\right)$ в ИК-спектре I регистрируются в сравнительно “открытой” части спектра в виде полосы сильной (при $668 \mathrm{~cm}^{-1}$ ) интенсивности.

Таким образом, продуктом взаимодействия в растворах $\mathrm{SO}_{2}-\mathrm{CH}_{3} \mathrm{NH}_{2}-\mathrm{H}_{2} \mathrm{O}-\mathrm{O}_{2}$ является сульфат метиламмония $\left(\mathrm{CH}_{3} \mathrm{NH}_{3}\right)_{2} \mathrm{SO}_{4}$, трехмерная структура которого стабилизирована системой Н- 
Таблица 4. Волновые числа $\left(\mathrm{cm}^{-1}\right)$ максимумов полос поглощения в ИК-спектре I

\begin{tabular}{|c|c|}
\hline ИК-спектр & Отнесение* \\
\hline $\begin{array}{l}3372 \mathrm{cp} . \\
3263 \mathrm{cp} .\end{array}$ & \\
\hline $3023 \mathrm{c}$. & $v_{\mathrm{as}, \mathrm{s}}\left(\mathrm{NH}_{3}^{+}\right)$ \\
\hline 2879 c. & $v_{\mathrm{as}, \mathrm{s}}\left(\mathrm{N}-\mathrm{CH}_{3}\right), v\left(\mathrm{NH}_{3}^{+}\right)$ \\
\hline $\begin{array}{l}2783 \text { с. } \\
2597 \text { ср. } \\
2506 \text { сл. } \\
2360 \text { ср. }\end{array}$ & \\
\hline 2342 сл. & $v\left(\mathrm{NH}_{3}^{+}\right)$ \\
\hline $1635 \mathrm{c}$. & $\delta_{\mathrm{as}}\left(\mathrm{NH}_{3}^{+}\right)$ \\
\hline $1521 \mathrm{c}$. & $\delta_{\mathrm{s}}\left(\mathrm{NH}_{3}^{+}\right)$ \\
\hline 1466 c. & $\delta_{\mathrm{as}}\left(\mathrm{N}-\mathrm{CH}_{3}\right)$ \\
\hline 1424 сл. & $\delta_{\mathrm{s}}\left(\mathrm{N}-\mathrm{CH}_{3}\right)$ \\
\hline 1276 сл. & $\tau\left(\mathrm{NH}_{3}^{+}\right)$ \\
\hline 1239 сл. & \\
\hline 1057 оч.с. & $v_{\text {as }}\left(\mathrm{SO}_{4}^{2-}\right), v\left(\mathrm{CN}^{+}\right)$ \\
\hline 969 c. & $v_{\mathrm{s}}\left(\mathrm{SO}_{4}^{2-}\right), \rho\left(\mathrm{CH}_{3}\right)$ \\
\hline $668 \mathrm{c}$. & $\delta_{\mathrm{as}}\left(\mathrm{SO}_{4}^{2-}\right)$ \\
\hline
\end{tabular}

* Деформационные: $\delta$ - ножничные, $\tau-$ крутильные; $\rho$ - маятниковые.

связей типа $\mathrm{NH}$ … О при участии всех атомов водорода “ониевого” катиона.

\section{СПИСОК ЛИТЕРАТУРЫ}

1. Химический энциклопедический словарь. М.: Советская энциклопедия, 1983. С. 328.

2. Hughes E.W., Lipscomb W.N. // J. Am. Chem. Soc. 1946. V. 68. № 10. P.1970. DOI: 10.1021/ja01214a029.

3. Zviedre I.I., Belyakov S.V. // Russ. J. Inorg. Chem. 2009. V. 54 . № 9. P. 1390. DOI: 10.1134/ S0036023609090083.
4. Gabe E.J. // Acta Crystallogr. 1961. V. 14. № 12. P. 1296. DOI:10.1107/S0365110X6100382X.

5. Włodarczyk-Gajda B., Rafalska-Łasocha A., Łasocha W.// Powder Diffraction. 2007. V. 22. № 3. P. 241. DOI: 10.1154/1.2770748

6. Mylrajan M., Srinivasan T.K.K., Sreenivasamurthy G. // J. Cryst. Spectr. Res. 1985. V. 15. № 5. P. 493. DOI: $10.1007 /$ BF01171052.

7. Zanazzi P.F. // Acta Crystallogr. 1968. V. 24B. P. 499. DOI: $10.1107 / \mathrm{S} 0567740868002724$.

8. Conley B.D., Yearwood B.C., Parkin S., Atwood D.A. // J. Fluor. Chem. 2002. V. 115. № 2. P. 155. DOI: 10.1016/S0022-1139(02)00046-5.

9. Vilminot S., Cot L., Avinens C., Maurin M. // Mat. Res. Bull. 1971. V. 6. P. 189.

10. Климова В.А. Основные методы анализа органических соединений. М.: Химия, 1975. 104 с.

11. Sheldrick G.M. // Acta Crystallogr., Sect. A. 2008. V. 64. P. 112.

12. http://webbook.nist.gov/cgi/cbook.cgi? ID $=$ C74895\& Units=SI\&Mask=200\#Mass-Spec.

13. Вульфсон Н.С., Заикин В.Г., Микая А.И. Масс-спектрометрия органических соединений. М.: Химия, 1986. C. 128.

14. Baek S.J., Choi K.-W., Choi Y.S., Kim S.K. // J. Chem. Phys. 2003. V. 118. № 24. P. 11040 . DOI: 10.1063/1.1575735.

15. Grossman S., Danielson N.D. // J. Chromatogr. A. 2009. V. 1216. № 16. P. 3578. DOI: 10.1016/j.chroma. 2008.09.064.

16. Преч Э., Бюльманн Ф., Аффольтер К. Определение строения органических соединений. Таблицы спектральных данных. Пер. с англ. М.: Мир; БИНОМ. Лаборатория знаний, 2006. 438 с.

17. Тарасевич Б.Н. ИК-спектры основных классов органических соединений. Справочные материалы. М.: МГУ, 2012. 54 с.

18. Накамото К. ИК-спектры и спектры КР неорганических и координационных соединений. М.: Мир, $1991.536 \mathrm{c}$. 